\title{
Necesidad de evaluación de la patología dual en contexto penitenciario
}

\section{Need for the assessment of dual diagnosis in prisons}

\author{
María José Casares-López*; Ana González-Me- \\ NÉNDEZ ${ }^{\star}$; María TeRESA Bobes-Bascarán ${ }^{\star *}$; RoBerto \\ SeCADES ${ }^{\star},{ }^{* \star *}$; Andrés Martínez-CORDERO ${ }^{\star \star *}$; Julı \\ BOBES $^{\star * * *}$
}

\author{
* Grupo de Investigación en Conductas Adictivas. Departamento de \\ Psicología. Universidad de Oviedo. \\ ** Hospital Clínico de Valencia - CIBERSAM. \\ *** Centro Penitenciario de Villabona (Asturias). \\ ${ }^{* * * *}$ Departamento de Medicina. Universidad de Oviedo. CIBERSAM.
}

\author{
Enviar correspondencia a: \\ María José Casares López \\ Grupo de Investigación en Conductas Adictivas. Departamento de \\ Psicología. \\ Universidad de Oviedo. Plaza Feijoo s/n, 33033 Oviedo \\ casaresmaria@uniovi.es
}

\section{RESUMEN}

Introducción: Los datos epidemiológicos recientes indican que un 92.5\% de los internados en prisión declaran haber consumido sustancias psicoactivas. A pesar de ellos, solamente un $2 \%$ de las investigaciones en patología dual se han realizado en el contexto penitenciario. Por ello, el objetivo del presente estudio descriptivo de carácter transversal, fue analizar los perfiles de patología dual en una prisión española y probar la factibilidad de dos instrumentos de evaluación.

Método: Se cuenta con una muestra de 152 internos procedentes de los módulos ordinarios del Centro Penitenciario de Villabona que manifestaron consumos compatibles con los criterios de un Trastorno por Uso de Sustancias y accedieron a realizar la entrevista voluntariamente. Se administraron la sexta versión del Índice de Gravedad de la Adicción-ASI-6- y la Entrevista Neuropsiquiátrica Internacional-MINI-.

Resultados: El perfil del entrevistado responde a un varón de 34 años, soltero, con hijos y con un tiempo medio de encarcelación que ronda los cinco años. Únicamente el $4.5 \%$ de los entrevistados declara no consumir drogas en la actualidad, llevando más de un año abstinente. De los consumidores en activo, la sustancia de consumo principal es mayoritariamente cocaina $(37,6 \%)$, heroina $(29,9 \%)$ o alcohol $(10,8 \%)$. El 52,7\% reconoce haber consumido en el último mes. Con respecto al estado psicopatológico, únicamente el 12,9\% no presenta patologías asociadas. Entre ellos, la sintomatología más prevalente es el trastorno antisocial de la personalidad (TAP, 65,6\%), riesgo de suicidio (45,2\%), depresión $(35,9 \%)$ y ansiedad $(25,5 \%)$.

Conclusión: Los instrumentos propuestos -ASI-6 y MINI- son pruebas factibles y recomendables para detectar la gravedad de la adicción y de la psicopatología asociada en este contexto.

Palabras clave: patología dual, trastornos relacionados con sustancias, diagnóstico, prisiones. recibido: Junio 2010 aceptado: Diciembre 2010

\section{ABSTRACT}

Introduction: Data show that $92.5 \%$ of prison inmates report drug or alcohol use. In spite of this, only $2 \%$ of dual diagnosis research has been carried out in the prison context. Therefore, the aim of this descriptive cross-sectional study was to analyze the profiles of dual diagnosis in a Spanish prison and test the feasibility of two assessment instruments. Method: The sample was made up of 152 drugaddicts imprisoned in the Villabona Penitentiary Center (Asturias, Spain) who volunteered to be interviewed. The sixth version of the Addiction Severity Index and the International Neuropsychiatric Interview -MINI- were used for the assessment of inmates' psychopathological status and drug problems. Results: The results show that, in general, the offender's profile is: male, 34 years old, unmarried, with children and with an average time spent in prison of approximately five years. Only $4.5 \%$ of respondents did not use drugs at the time of the study. In the remainder, the most widely used substances are cocaine (37.6\%), heroin (29.9\%) and alcohol (10.8\%), with $52.7 \%$ reporting having used drugs in the last month. With regard to psychopathological state, only $12.9 \%$ have no associated disorders, and the most prevalent symptoms correspond to antisocial personality disorder (65.6\%), risk of suicide (45.2\%), depression (35.9\%) and anxiety (25.5\%).

Conclusion: The instruments proposed (ASI-6 and MINI) are feasible tools for detecting addiction severity and associated psychopathology in this context.

Key words: dual diagnosis, substance-use disorders, diagnosis, prison. 


\section{INTRODUCCIÓN}

- xiste un conocido ciclo de consumo de drogas y comisión de delitos. Según datos de la Universidad de - Pensilvania (comunicación personal), el 80\% de los internados en prisión han cometido delitos relacionados con las sustancias, y son consumidores habituales. Las drogas y/o el alcohol, estuvieron envueltos en el $50 \%$ de los delitos con violencia, 50\% maltrato doméstico, 80\% maltrato infantil, entre el 50-70 \% de los delitos contra la propiedad y entre el 75-99\% de los delitos por tráfico de sustancias. En España, en 1998 se calculó que más del 50\% la población que ingresa en prisión presentó problemas de consumo de sustancias psicoactivas (Observatorio Español sobre Drogas, 1999). Estos datos coinciden con los ofrecidos por estudios europeos, en los que se afirma que aproximadamente la mitad de la población de reclusos son dependientes de las drogas, principalmente de opioides, cannabis y alcohol (Marshall, Simpson y Stevens, 2000).

En los últimos años, la cocaína ha relegado el consumo de heroína a un segundo plano, tanto en cárceles como en la población general (EDADES, 2008, ESDIP, 2006). En la última encuesta penitenciaria (ESDIP, 2006) se reveló que el 92,5\% de los entrevistados había consumido alguna vez en la vida al menos una de las sustancias por las que se preguntaba (incluido alcohol). Sólo el 7,5\% de la muestra manifestó no haber consumido a lo largo de su vida ninguna sustancia psicoactiva (no incluido tabaco). Las mayores prevalencias de consumo (alguna vez en la vida) se dan para el alcohol $(88,3 \%)$, el cannabis $(64,8 \%)$ y la cocaína en polvo $(53,3 \%)$. Esta misma fuente permite conocer datos de consumo dentro de la cárcel en el último mes, principalmente de psicofármacos y cannabis $(28,5 \%)$, cocaína $(3,4 \%)$, heroína $(2,9 \%)$, y también, que el último mes en libertad antes del ingreso en IIPP el consumo era más frecuente: alcohol $(22,7 \%)$, cocaína $(19,2 \%)$ y mezcla de heroína y cocaína $(14,5 \%)$.

Hoy en día, la patología dual (PD), es una realidad clínica a tener en cuenta por todos los profesionales que trabajan en el ámbito clínico; puesto que el uso de sustancias está fuertemente asociado con la morbilidad psiquiátrica (Barea et al, 2002; Casas y Guardia, 2010). En las últimas décadas, mediante estudios epidemiológicos, se ha determinado la relación entre las conductas adictivas y otros trastornos psicológicos (Compton, Thomas, Conway y Colliver, 2005).

Estudios epidemiológicos sobre la prevalencia de PD en población general, como el ECA (Regier et al., 1990), el NLAES (NIAAA, 1998) o el NCSR (Kessler et al., 2005; Kessler y Merikangas, 2004) obtienen datos de prevalencia que oscilan entre un $47,7 \%$ y un $83,6 \%$ de comorbilidad de trastornos por uso de sustancias -TUS- con Trastornos de Personalidad. Respecto a la morbilidad entre TUS y trastornos afectivos oscila entre el 32 y el $20,13 \%$, y trastornos de ansiedad entre el $23,7 \%$ al 17,75\%, respectivamente. Puede advertirse que en el estudio de Kessler y Merikangas (2004), se obtienen tasas de prevalencia más bajas con respecto a aquellos realizados en los años 90 .
Otros, han llevado a cabo estudios sobre tratamientos específicos como Programas de Mantenimiento con Metadona (Cacciola, Alterman, Rutherford, McKay y Mulvaney, 2001; Gelkopf, Weizman, Melamed, Adelson y Bleich, 2006) y encuentran datos que van desde un $24 \%$ de comorbilidad con trastornos del Eje I a lo largo de la vida, hasta un $82,8 \%$ en este período temporal, o 66,2 \% de coexistencia actual; o en tratamientos ambulatorios para adicción al alcohol, donde Pettinati, Rukstalis, Luck, Volpicelli y O'brien (2000) examinaron 196 pacientes, de los cuales más de un tercio (el 36,2\%) presentan un trastorno del Eje I comórbido en la actualidad. La mayoría de ellos padecen un trastorno depresivo (77\%).

Asimismo, la comorbilidad de trastornos afectivos y TUS en población consumidora de heroína se sitúa entre el 12 y el $88 \%$ en una cohorte de sujetos entre 18 y 30 años (RodríguezLlera et al., 2006).

Como hemos podido contemplar, la prevalencia de patología dual se ha estudiado en diversos contextos, principalmente en los sistemas de tratamiento para el abuso de sustancias y en los diferentes sistemas asistenciales, sin embargo, el contexto penitenciario es el ambiente que menos atención recibe, a pesar de ser una oportunidad única para la intervención y acoger un porcentaje ingente de reclusos drogodependientes.

Con todo, Fazel y Danesh (2002) reclaman la necesidad de estudios en profundidad en población penitenciaria, pues consideran que a pesar de las numerosas investigaciones desarrolladas, éstas ordinariamente se realizan con muestras muy pequeñas y una población preseleccionada (p.ej. del Servicio Psiquiátrico) y adolecen en general de una evaluación sistemática. Los autores revisaron un total de 66 publicaciones en las que se analizan 22790 reclusos. Encontraron una prevalencia de 3,7 \% en trastornos psicóticos, de $10 \%$ en depresión mayor y de un 65\% en trastornos de personalidad; de los que el $48 \%$ se trataba de un trastorno de personalidad antisocial. Todo esto indica que el riesgo de padecer un trastorno psicótico o depresivo es entre 2-4 veces mayor en población encarcelada que en la población general.

Sin embargo, ello no ha llevado a que el interés por el estudio de la patología dual se haya despertado en instituciones penitenciarias. Aunque las tasas de prevalencia varian, los resultados indican que los reclusos con patología dual representan una parte significativa de la población penitenciaria. En los años 90, los porcentajes oscilaban entre el 10 y el $23 \%$ de patología dual en reclusos (Edens, Peters y Hills, 1997; National GAINS Center, 1999; Swartz y Lurigio, 1999), pero en el siglo XXI aumenta el interés y la conciencia de este fenómeno, y las tasas de prevalencia aumentan a un rango del 20 al 50 \% (Lapham et al., 2001; Peters, LeVasseur y Chandler, 2004; Smith, Sawyery Way, 2002).

Los autores Cacciola y Dugosh (2003) conscientes de que la patología dual afecta a millones de personas en EEUU, realizan una revisión de la literatura que aborda la investigación en la comorbilidad con TUS entre la población general y entre individuos en una variedad de sistemas de tratamiento. La atención se centró en los estudios más recientes a su fecha de realización (1997-2003) con las muestras 
procedentes de los Estados Unidos, pero incluyeron estudios anteriores para contextualizar los resultados. Si bien hubo importante variabilidad en las tasas de comorbilidad entre los estudios, se suelen tratar más la patología dual en los sistemas de salud mental que en los programas para el abuso de sustancias. Categorizaron los estudios según el contexto, concretamente, para el tratamiento de las toxicomanías contemplaron tratamiento ambulatorio, mantenimiento de metadona, residenciales, etc. Sin embargo, solamente 6 investigaciones se realizaron en contexto penitenciario, lo que representa un 2\% del total. El resto, se distribuyen como sigue: $31 \%$ en modalidad de tratamiento ambulatorio, $26 \%$ en unidades de hospitalización, $17 \%$ en múltiples modalidades, $9 \%$ en tratamiento residencial, 7\% en tratamiento sustitutivo y un $7 \%$ no especificado en el estudio. Estos estudios llevados a cabo en prisión acerca de la PD se centran en datos de prevalencia y perfiles de los internos con comorbilidad, detectando prevalencias altas en centros penitenciarios de Trastorno de Personalidad Antisocial junto con Trastornos Depresivos (Pelissier y O'Neil, 2000) y Ansiosos (White, Goldkamp y Campbell, 2006). El estudio de Harris y Koepsell (1998) se centra por otro lado en las tasas de reencarcelación de reclusos con y sin PD. A los 12 meses, no se encuentran diferencias estadísticamente significativas entre reclusos con/sin $\mathrm{PD}$, el 54,3\% del grupo de pacientes duales y 51,2 por ciento del grupo sin PD volvieron a ser detenidos. Por último, del estudio de Edens, Peters y Hills (1997) se deducen modificaciones importantes a tener en cuenta en el tratamiento intrapenitenciario de reclusos con PD, como sería un mayor apoyo individual, menor ratio staff-paciente, disminución de la confrontación, y formación continuada del personal que administra los programas.

En un reciente estudio llevado a cabo en nuestro país, Casares-López et al. (2010) encuentran una alta tasa de trastornos de personalidad en una muestra de 76 reclusos que decidieron ingresar en una unidad libre de drogas intrapenitenciaria voluntariamente a lo largo del año 2006. Los porcentajes de PD más altos se obtuvieron en el área de abuso de alcohol, donde el $48,7 \%$ de la muestra presenta este trastorno, así como en el patrón de personalidad antisocial y dependiente $(47,4 \%$ y $42,7 \%$, antisocial y dependiente respectivamente), evaluados a través del Inventario MCMI-II (Millon, 1997). En cuanto a los síndromes graves, el 39,5\% de los casos puede recibir el diagnóstico de trastorno delirante, el $26,7 \%$ de trastorno del pensamiento del tipo psicótico y por último el 23\% de los casos encajarian en el diagnóstico de depresión mayor, con una Tasa Base $\geq 75$ en el MCMI-II.

Solamente un 2\% de los estudios sobre PD se realizan en prisión, por lo que no existen en la literatura referencias acerca de las propiedades psicométricas de instrumentos de evaluación psicológica, medio primordial para poder cumplir con el Artículo $2^{\circ}$ del Reglamento Penitenciario de 1996, que establece como fin principal de la actividad penitenciaria la reeducación y reinserción social de los sentenciados a penas y medidas de seguridad privativas de libertad, así como la retención y custodia de los detenidos, presos y penados, y la asistencia social a los internos, a los liberados y a sus familiares.
Por lo tanto, el objetivo del presente trabajo descriptivo fue conocer el estado psicopatológico de los internos del centro penitenciario de Villabona con TUS, la frecuencia de PD y las variables intervinientes en su desarrollo y comprobar la viabilidad (feasibility) de los instrumentos utilizados para detectar la gravedad de la adicción y la psicopatología asociada en este contexto.

\section{MATERIAL Y MÉTODO}

\section{Muestra}

La muestra final la formaron un total de 149 internos en régimen de segundo y tercer grado en el Centro Penitenciario de Villabona $(n=107)$ y el Centro de Inserción Social, CIS "El Urriello" ( $n=42)$, ambos en Asturias, España. El régimen de segundo grado se caracteriza por el imperio de los principios de seguridad, orden y disciplina mientras que el régimen abierto se aplica a los reos clasificados en tercer grado, y en este caso se cumple en un centro abierto. El porcentaje de varones fue del $95.3 \%$ y la edad media unos 35 años. Todos ellos, accedieron voluntariamente a la realización de una entrevista con personal entrenado en el uso de los instrumentos aplicados, y para su inclusión en el estudio se observó que cumplieran criterios de dependencia de sustancias actual o reciente, y de la misma forma, firmaron el consentimiento informado por escrito.

\section{Instrumentos de medida}

Addiction Severity Index-6 (ASI-6) (Bobes et al., 2007): EI ASI-6 es una entrevista semiestructurada y heteroaplicada que valora multidimensionalmente la gravedad de la adicción, entendida esta como necesidad de tratamiento. EI ASI-6 ofrece perfil de gravedad de cada paciente en diferentes áreas de la vida del sujeto (estado de salud orgánica, mental, situación legal, familiar, social, etc.), y ha sido adaptado a población española recientemente (Díaz-Mesa et al., 2010).

Para evaluar la comorbilidad con psicopatología del Eje I y el riesgo de suicidio se utilizó la adaptación española (Ferrando,Bobes, Gibert y Lecrubier, 1997) de la MINI-Entrevista Neuropsiquiátrica Internacional (Sheehan et al., 1998). La versión actual, MINI 4.6, rastrea de forma estandarizada los criterios necesarios para realizar diagnósticos de acuerdo al DSM-IV/ CIE-10. Se trata de una entrevista diagnóstica altamente estructurada, relativamente breve y de fácil utilización. La entrevista está dividida en 14 secciones diagnósticas, entre las que se encuentra una sección correspondiente al consumo excesivo de alcohol. Al inicio de cada sección existe un apartado de screening que permite, en caso de no cumplir los criterios, pasar a la sección diagnóstica siguiente.

\section{Procedimiento}

La administración de las entrevistas se realizó de modo individual y heteroaplicado, invirtiendo para ello una media 
de 90 minutos por persona, en las instalaciones facilitadas por el centro penitenciario.

\section{Análisis de datos}

Se llevaron a cabo diferentes análisis de frecuencias para describir las características de la muestra total y para obtener el perfil de paciente de cada uno de los grupos. Se utilizó el programa estadístico SPSS-15. Mediante la estadística bivariada, se analiza la relación entre variables psicopatológicas, variables de gravedad de la adicción y otras variables personales (Chi Cuadrado - $\chi^{2}$ - con corrección de Fisher o de Yates).

\section{RESULTADOS}

El perfil del recluso del centro penitenciario es varón, con una edad media de 34,21 años, nunca casado $(51,4 \%$ ) y con hijos (56,3\%). El 39.2\% ha cursado los estudios básicos, existiendo un $37,2 \%$ sin estudios ni titulación académica alguna. En cuanto al estado de salud, existe un alto porcentaje de personas con procesos infecciosos crónicos (ver Tabla 1).

El 4,5\% de los entrevistados reconoce llevar más de un año abstinente. Aunque la mayoría se declara policonsumidor, la sustancia principal de consumo en esta muestra es la cocaína (ver Tabla 1). Un 46,8\% de ellos declara haber conseguido mantenerse abstinente al menos un año a lo largo de su vida, habiendo recibido algún tipo de tratamiento para su adicción el 70,0 \%. El 46,3\% han utilizado la vía de administración intravenosa en alguna ocasión, y un 55,1\% reconoce abiertamente haber consumido en el último mes. La edad media de inicio de consumo de la sustancia principal se sitúa en 17,53 años, y han consumido de media 5 sustancias por persona $(5,43)$. En el círculo cercano familiar y/o social, reconocen tener personas que son también consumidoras un $60,2 \%$.

Tabla 1: Consumo de sustancias y enfermedades crónicas $(n=$ 143 reclusos con TUS)

\begin{tabular}{lcc}
\hline & Sustancia problema principal & \\
\hline Heroina & Frecuencia & Porcentaje \\
Cocaina & 47 & 32,9 \\
Cannabis & 59 & 41,3 \\
Alcohol & 15 & 10,5 \\
Anfetaminas & 17 & 11,9 \\
Psicofármacos & 2 & 1,4 \\
Alucinógenos & 1 & 0,7 \\
\hline & 2 & 1,4 \\
\hline VHC & Enfermedades crónicas & \\
VIH & 65 & 45,8 \\
Cirrosis & 9 & 6,3 \\
Otras & 7 & 4,9 \\
\hline
\end{tabular}

A propósito de la situación legal, el número medio de detenciones es 19.59 por persona, y el número medio de condenas es 4,53, con un tiempo de encarcelación a lo largo de la vida de unos cinco años (63,04 meses). El 81,7\% se encuentra en segundo grado penitenciario, y un 17,1\% en tercer grado. A una persona no se le había asignado grado penitenciario aún al estar en situación preventiva, esto es, pendiente de juicio. La primera detención se produjo a los 20,87 años de media, y la primera sentencia firme se produjo a los 23,98 años. El primer acto delictivo cometido, algunos siendo menores de edad, fueron delitos contra la propiedad $(44,4 \%)$, seguido de delitos violentos, como lesiones, agresiones o robos con violencia $(20,0 \%)$ o delitos de posesión o tráfico de drogas (7,8\%). El delito por el que cumplen condena actualmente es principalmente del tipo "delitos contra la propiedad" $(31,5 \%)$, seguido de tráfico de drogas $(16,3 \%) 0$ delitos violentos $(9,8 \%)$.

Un porcentaje del $33,1 \%$ ha sido tutelado por los Servicios de Protección del Menor con internamiento en un centro de menores. El 39,6\% ha tenido algún familiar cercano ingresado en prisión previamente. Cabe destacar que un $34,7 \%$ refiere haber sido víctima de malos tratos físicos en la niñez, un 33,7\% malos tratos psicológicos, y un 4,1 \% ha sufrido abusos sexuales. En el 33,3\% de los casos, el agresor era una persona familiar o conocida para el niño. A lo largo de la vida, el 66,9\% manifiestan que han sufrido una situación traumática en la vida en la que había temido por su vida (accidente, incendio, tiroteos...).

Con respecto al estado psicopatológico, solamente el $35,4 \%$ de la muestra reconoce tener algún diagnóstico psiquiátrico ya establecido, aunque cabe destacar que un 38,5 $\%$ no son conocedores del contenido de sus informes psi-

Tabla 2: Tasas de presencia de sintomatología psiquiátrica

\begin{tabular}{lcc}
\hline Tipo de trastorno & Frecuencia & Porcentaje \\
\hline Trastorno antisocial de la personalidad-TAP- & 100 & 86,2 \\
Riesgo de suicidio & 61 & 52,6 \\
Trastorno depresivo & 50 & 43,1 \\
Trastorno de ansiedad generalizado -TAG- & 37 & 31,9 \\
Agorafobia & 24 & 20,7 \\
Trastorno por estrés post-traumático -TEPT- & 15 & 12,9 \\
Trastorno psicótico & 15 & 12,9 \\
Episodio maniaco actual & 14 & 13,5 \\
Trastorno Distimico & 11 & 9,5 \\
Trastorno de angustia & 10 & 7,5 \\
Fobia social & 9,6 & 7,8 \\
\hline Trastorno obsesivo-compulsivo -TOC- & 11 & 9,5 \\
\hline
\end{tabular}


Tabla 3: Asociación entre maltrato infantil, patología dual y riesgo de suicidio

\begin{tabular}{|c|c|c|c|c|}
\hline & \multicolumn{3}{|c|}{ Patología dual $\mathrm{f}(\%)$} & \multirow{2}{*}{$\chi^{2}$} \\
\hline & & No & $\mathrm{Si}$ & \\
\hline & No & $50(79,4)$ & $13(20,6)$ & $\chi^{2}(1, n=61)=15,68$ \\
\hline \multirow[t]{2}{*}{ Maltrato fisico en la infancia } & Si & $11(35,5)$ & $20(64,5)$ & p<,001, Phi<,001 \\
\hline & No & $51(81,0)$ & $12(19,0)$ & $\chi^{2}(1, n=61)=19,13$ \\
\hline \multirow[t]{4}{*}{ Maltrato psicológico en la infancia } & Si & $11(35,5)$ & $20(64,5)$ & p<,001, Phi<,001 \\
\hline & \multicolumn{3}{|c|}{ Riesgo de Suicidio f (\%) } & \\
\hline & & No & Si & \\
\hline & No & $38(80,9)$ & $9(19,1)$ & $\chi^{2}(1, n=61)=9,15$ \\
\hline \multirow[t]{2}{*}{ Maltrato fisico en la infancia } & $\mathrm{Si}$ & $23(48,9)$ & $24(51,1)$ & $p=, 002, P h i=, 001$ \\
\hline & No & $38(80,9)$ & $9(19,1)$ & $\chi^{2}(1, n=62)=8,00$ \\
\hline Maltrato psicológico en la infancia & $\mathrm{Si}$ & $24(51,1)$ & $23(48,9)$ & $p=, 005$, Phi $=, 002$ \\
\hline
\end{tabular}

quiátricos o no lo entienden. Un 40,0 \% reciben tratamiento psicofarmacológico para el mismo, ya prescrito antes del ingreso en prisión en el 36,1\% de los casos. El 4,5\% reconocen que en su expediente consta un diagnóstico de trastorno de ansiedad, el 16,9\% de trastorno psicótico, 4,5\% trastornos depresivos, 6,7\% trastornos de personalidad (no especificados por el paciente), 1,1\% trastorno de la identidad sexual y $1,1 \%$ discapacidad intelectual.

Un 12,9\% no tienen ningún trastorno actual siguiendo los criterios de la MINI $(n=21)$, mientras que un $17,7 \%$ de la muestra cumple los criterios DSM-IV para un TUS, pero no presenta otro trastorno comórbido ( $n=25$ personas). Del $82,3 \%$ restante, se detecta sintomatología para 4,32 trastornos evaluados de media, aunque hay que tener en cuenta los solapamientos, puesto que evalúa varios trastornos depresivos, varios de ansiedad, y en ocasiones episodios pasados y actuales. Entre ellos, los más prevalentes podemos contemplarlos en la Tabla 2.

Los análisis $\chi^{2}$ de independencia (con corrección de Yates) indican una asociación significativa entre la presencia de historia de maltrato físico o psicológico en la infancia y el desarrollo posterior de psicopatología. En la Tabla 3 puede contemplarse la influencia de estas variables personales en la presencia en la actualidad de patología dual y en el riesgo de suicidio.

\section{DISCUSIÓN}

Los resultados indican que un alto porcentaje de las personas internadas en prisión presentan un trastorno de consumo de sustancias unido a otros trastornos mentales. El $78,7 \%$ de la muestra presenta un trastorno comórbido al TUS, resultados que superan a los ofrecidos por Piselli, Elisei, Murgia, Quartesan y Abram (2009) para quienes uno de cada cinco reclusos $(20,9 \%)$ tenían comorbilidad por uso de sustancias y trastornos psiquiátricos concordando con otros muchos, coincidiendo por ejemplo, con la tasa de patología dual del $80 \%$ en personas encarceladas, frente al $31 \%$ observado en la comunidad, obtenidas por Butler et al. (2007) o con los hallazgos de Gunter et al. (2008), para quienes más del 90 por ciento de los reclusos cumplian los crite- rios de un trastorno psiquiátrico actual o pasado, siendo los más frecuentes los trastornos por uso de sustancias (90\%), trastornos del humor (54\%), trastornos psicóticos (35\%), trastorno de personalidad antisocial (35\%) con una media de 4,2 trastornos (dos tercios tenían 3 o más trastornos). Estos resultados en un centro penitenciario español superan también las tasas de pacientes de centros de salud mental y centros asistenciales de la Comunidad de Madrid, para los que el 53,2\% de los pacientes 837 casos de la muestra cumplen los criterios diagnósticos de Patología Dual, siendo los trastornos mentales más frecuentes los trastornos de personalidad (71\% de los pacientes), acompañados la mayoría de las veces con riesgo de suicidio $(41,4 \%)$, episodio (hipo) maniaco $(31,5 \%)$, trastorno de angustia $(29,0 \%)$, episodio depresivo mayor $(28,8 \%)$, trastorno de ansiedad $(25,2 \%)$ y trastorno distímico (22,9\%) (AEPD, 2008).

Es importante la evaluación del trastorno de personalidad antisocial en entornos correccionales, puesto que destaca por su fuerte asociación con el delito, y en particular, el delito violento. Por ejemplo, los individuos con TAP cometen de 12 a 16 veces más actos violentos que sujetos con esquizofrenia o trastorno bipolar (Swanson, Holcer, Ganju y Jono, 1990). La importancia del diagnóstico de los pacientes duales con TAP radica en la dificultad de las terapias grupales, puesto que estos pacientes integrados en grupos con otras patologías, habitualmente perjudican el tratamiento de los demás miembros, con actitudes manipuladoras, rentistas y descalificadoras (Garrido y Dorado, 2010).

La prevalencia de patología dual en prisión, como hemos visto, es muy superior a la existente en la población general, considerando que al menos el 25,6\% de los internos en 2006 presentan uno o más diagnósticos psiquiátricos graves reflejados en su Historia Clínica, y llegando al 49,6\% si consideramos antecedentes de abuso de drogas, así como un 12,1\% con PD. (IIPP, 2007; Fanzel y Dasen; 2002).

Estudios sobre la salud mental de los internados en prisión ofrecen tasas menores utilizando el mismo instrumento diagnóstico (MINI), por lo que podemos suponer que no sólo es más alto el porcentaje de trastornos psicopatológicos en prisión, sino que aumenta aún más cuando se unen las condiciones cárcel más TUS. Los datos de Fotiadou, Livaditis, Manou, Kaniotou y Xenitidis (2006) muestran una prevalen- 
cia del $78.7 \%$ de trastornos psicopatológicos. Los principales diagnósticos fueron: trastorno de ansiedad (37,5\%), al igual que en esta muestra, es prioritaria la sintomatología ansiosa, viéndose incrementada cuando se une a un TUS (42,3\%); seguido de depresión (27,5\%), en nuestra muestra el 38,9\% presenta síntomas afectivos, y trastorno de personalidad antisocial (37,5\%), 86,2\% en nuestra muestra. En el estudio de Fontiadou et al. (2006) un 11,2\% se diagnostica con un trastorno bipolar o esquizofrénico mientras el 28,2\% de los pacientes con comorbilidad muestra sintomatología psicótica. El único trastorno que se ha revelado con un mayor porcentaje en la muestra sin TUS del estudio de 2006, ha sido el retraso mental, que aparece en un 15\% de internos con un $\mathrm{Cl}$ inferior a 75, mientras en el presente estudio solamente una persona cumple este criterio.

Sin embargo, los reclusos que reciben tratamiento para su TUS son relativamente pocos, y las intervenciones existentes tienden a ser a corto plazo o no clínicas, por lo que se necesita una adaptación del nivel de atención con las necesidades reales (Belenko y Peugh, 2005). Las dimensiones adicionales del abuso de drogas y sus efectos deben evaluarse para hacer clínicamente estimaciones adecuadas de necesidad de tratamiento y la elaboración de planes de tratamiento apropiados (Carise, Gurel, Kendig y McLellan, 2002; Simpler y Langhinrichsen-Rohling, 2005), por lo que la investigación en este campo es trascendental. Tampoco existe un gran volumen de literatura que se ocupe del análisis de los factores intervinientes en el desarrollo de patología dual en prisión. En el presente estudio se observa una alta asociación entre el maltrato infantil y la patología dual o el riesgo de suicidio. Los futuros pasos de esta investigación serán precisamente intentar dilucidar qué variables influyen en el desarrollo de la patología dual en población penitenciaria, y comprobar si existe posibilidad de intervención en alguna de ellas para lograr reducir, por ejemplo, las conductas autolíticas.

En conclusión, la frecuencia de PD en prisión es un problema a tratar, que necesita evaluación y tratamientos eficaces para lograr la rehabilitación y reinserción de los internos. Los instrumentos utilizados en el presente estudio resultaron ser pruebas factibles para detectar la gravedad de la adicción y necesidad de tratamiento, así como la psicopatología asociada en el contexto penitenciario, puesto que proporcionan una evaluación multidimensional en una sola sesión, con un alto grado de sistematización, detectando también el riesgo de comisión de una tentativa suicida. Asimismo, la MINI ha sido ya utilizada en varias investigaciones en contexto penitenciario (Easton, Devine, Scott y Wupperman, 2008; Fotiadou, Livaditis, Manou, Kaniotou y Xenitidis, 2006; Gunter et al., 2008), obteniendo resultados muy similares y coherentes con el perfil de población atendida, incluyendo trastornos del Eje I y el trastorno de personalidad antisocial, muy prevalente en centros penitenciarios. Por supuesto, la evaluación de los trastornos del Eje II es también un aspecto a añadir, puesto que el instrumento MINI solamente explora el TAP. Esto no exime de la necesidad de otros instrumentos diagnósticos para confirmar las sospechas detectadas con los instrumentos de screening, y una formación exhaustiva del personal para su aplicación.

\section{AGRADECIMIENTOS}

Este trabajo ha sido financiado por la ayuda predoctoral "Severo Ochoa", BP07-095; otorgada por la Fundación para el Fomento en Asturias de la Investigación Científica Aplicada y la Tecnología -FICYT-.

\section{REFERENCIAS}

Asociación Española de Patología Dual -AEPD- (2008). Estudio epidemiológico para determinar la prevalencia diagnostico y actitud terapéutica de la patología dual en la Comunidad de Madrid. . Recuperado el día 4 de Noviembre de 2010 de http:// www.madridsalud.es/noticias/Presentacion_espad_nov08.pdf.

Barea, J., Benito, A., Real, M., Mateu, C., Martín, E., López, N. y Haro, G. (2010). Estudio sobre aspectos etiológicos de la patología dual. Adicciones, 22, 15-24.

Belenko, S. y Peugh, J. (2005). Estimating drug treatment needs among state prison inmates. Drug and Alcohol Dependence, 77, 269-281.

Bobes, J., Bascarán, M. T., Bobes-Bascarán, M. T., Carballo, J. L., Díaz Mesa, E. M., Flórez, G., et al. (2007). Valoración de la gravedad de la adicción: aplicación a la gestión clínica y monitorización de los tratamientos. Madrid: Delegación del Gobierno para el Plan Nacional sobre Drogas.

Butler, T., Andrews, G., Allnutt, S., Sakashita, C., Smith, N. E. y Basson, J. (2006). Mental disorders in Australian prisoners: a comparison with a community sample. Australian and New Zealand Journal of Psychiatry, 40, 272-276.

Cacciola, J.S. y Dugosh, K. (2003). Co-occurring substance use and mental disorders: an annotated bibliography. Recuperado el dia 4 de Noviembre de 2010 de: http://tie.samhsa.gov/Topics/Pdf/ annotatedbibliography.pdf

Cacciola, J.S., Alterman, A.I., Rutherford, M.J., McKay, J.R. y Mulvaney, F.D. (2001). The relationship of psychiatric comorbidity to treatment outcomes in methadone maintained patients. Drug and alcohol dependence, 61, 271-80.

Carise, D., Gurel, O., Kendig, C. y McLellan, A. T. (2002). Giving Clinical Meaning to Patient Assessment: Technology Transfer to Improve Treatment Care Planning and Service Delivery. Philadelphia, PA.: Treatment Research Institute.

Casares-López, M.J., González, A., Torres, M., Secades, R., FernándezHermida, J.R. y Álvarez, M. (2010). Comparación del perfil psicopatológico y adictivo de dos muestras de adictos en tratamiento: en prisión y en comunidad terapéutica. International Journal of Clinical and Health Psychology, 10, 225-243.

Casas, M. y Guardia, J. (2002). Patología psiquiátrica asociada al alcoholismo. Adicciones, 14, 195-219.

Compton, W. M., Thomas, Y. F., Conway, K. P., y Colliver, J. D. (2005). Developments in the epidemiology of drug use and drug use disorders. American Journal of Psychiatry, 162, 1494-1502.

Delegación del Gobierno para el Plan Nacional sobre Drogas (1999). Informe $n^{\circ} 2$ del Observatorio Español sobre Drogas. Recuperado el dia 4 de Noviembre de 2010 de http://www.pnsd. msc.es/Categoria2/publica/pdf/oed-2.pdf.

Delegación del Gobierno para el plan Nacional Sobre Drogas (2006). Encuesta sobre salud y consumo de drogas a los internados 
en instituciones penitenciarias. Recuperado el dia 9 de Julio de 2010 de http://www.pnsd.msc.es/Categoria2/publica/pdf/ encuestaPenitenciaria2006.pdf.

Delegación del Gobierno para el Plan Nacional sobre Drogas. (2008). Informe de la encuesta domiciliaria sobre alcohol y drogas en España (EDADES) 2007/08. Madrid: Ministerio de Sanidad y Consumo.

Díaz-Mesa, E.M., García-Portilla, P., Sáiz, P., Bobes, T., Casares, M.J., Fonseca, E., Carreño, E., Florez, G., Guardia, J., Ochoa, E., Pereiro, C., Rubio, G., Terán, A., Fernández-Hermida, J.R. y Bobes, J. (2010). Rendimiento psicométrico de la $6^{a}$ versión del Addiction Severity Index en español (ASI-6). Psicothema, 22, 513-519.

Dirección General de Instituciones Penitenciarias. Subdirección General de Sanidad Penitenciaria (2006). Estudio sobre Salud Mental en el medio penitenciario, Diciembre de 2006. Madrid: Subdirección General de Sanidad Penitenciaria.

Easton, C.J., Devine, S., Scott, M. y Wupperman, P. (2008). Commentary: Implications for Assessment and Treatment of Addictive and Mentally Disordered Offenders Entering PrisonsJournal of the American Academy of Psychiatry and the Law, 36, 35 - 37.

Edens, J. F., Peters, R. H. y Hills, H. A. (1997). Treating prison inmates with co-occurring disorders: An integrative review of existing programs. Behavioral Sciences \& the Law, 15, 439-457.

Ferrando, L., Bobes, J., Gibert, J. y Lecrubier, Y. (1997). Mini International Neuropsychiatric Interview. En: Bobes J, Bousoño M, González MP, editores. Manejo de los trastornos mentales y del comportamiento en Atención Primaria. 2a ed. Oviedo: Gofer; 1997.

Fanzel, S. y Dansen, J. (2002). Serious mental disorder in 23000 prisoners: a systematic review of 62 surveys. The Lancet, 16, 545-550.

Fotiadou, M., Livaditis, M., Manou, I., Kaniotou, E., y Xenitidis, K. (2006). Prevalence of mental disorders and deliberate selfharm in Greek male prisoners. International Journal of Law and Psychiatry, 29, 68-73.

Garrido, V. y Dorado, M. (2010). Trastorno antisocial de la personalidad y adicciones. En G. Haro, J. Bobes, M. Casas, J. Didia y G. Rubio (Eds.), Tratado sobre patología dual. Reintegrando la salud mental. Castellón: MRA.

Gelkopf, M., Weizman, T., Melamed, Y., Adelson, M. y Bleich, A. (2006). Does psychiatric comorbidity affect drug abuse treatment outcome? A prospective assessment of drug abuse, treatment tenure and infectious diseases in an Israeli methadone maintenance clinic. Israel Journal of Psychiatry \& Related Sciences, 43, 126-36.

González, F., Salvador, L., Martínez, J.M., López, A., Ruz Franzi I. y Guerra, D. (2002). Estudio de fiabilidad y validez de la versión española de la entrevista clínica Addiction Severity Index (ASI). En: I. Iraurgi Castillo, F. González Sáiz (Eds.). Instrumentos de evaluación en drogodependencias (pp. 271-307). Barcelona: Aula Médica.

Gunter, T. D., Arndt, S., Wenman, G., Allen, J., Loveless, P., Sieleni, B., Y Black, D. W. (2008). Frequency of mental and addictive disorders among 320 men and women entering the lowa prison system: use of the MINI-Plus. Journal of the American Academy of Psychiatry and the Law, 36, 27-34.

Harris, V., y Koepsell, T. D. (1998). Rearrest among mentally ill offenders. Journal of the American Academy of Psychiatry \& the Law, 26, 393-402.
Kessler, R. C., Birnbaum, H., Demler, O., Falloon, I. R., Gagnon, E., Guyer, M., Howes, M. J., Kendler, K. S., Shi, L., Walters, E. y Wu, E. 0. (2005). The prevalence and correlates of nonaffective psychosis in the National Comorbidity Survey Replication (NCS-R). Biological Psychiatry, 58, 668-676.

Kessler, R. C. y Merikangas, K. R. (2004). The National Comorbidity Survey Replication (NCS-R): background and aims. International Journal of Methods in Psychiatric Research, 13, 60-68.

Lapham, S. C., Smith, E., C'De Baca, J., Chang, I., Skipper, B. J., Baum, G. y Hunt, W. C. (2001). Prevalence of psychiatric disorders among persons convicted of driving while impaired. Archives of General Psychiatry, 58, 943-949.

Marshall, T., Simpson, S. y Stevens, A. (2000). Health Care in Prisons: A Health Care Needs Assessment. University of Birmingham. Recuperado el dia 6 de junio de 2010 de http://www.doh.gov.uk/ prisonhealth/prisons.htm.

Millon, T. (1997). Millon Clinical Multiaxial Inventory-II (MCMI-II). Minneapolis: National Computer Systems.

National GAINS Center. (1999). Drug courts as a partner in mental health and co-occurring substance use disorders diversion programs. Delmar, NY: National GAINS Center.

NIAAA. Drinking in the United States: Main Findings from the 1992 National Longitudinal Alcohol Epidemiologic Survey (NLAES). National Institute on Alcohol Abuse and Alcoholism; 1998. NIH Publication No. 99-3519.

Pelissier, B. M., y O'Neil, J. A. (2000). Antisocial personality and depression among incarcerated drug treatment participants. Journal of Substance Abuse, 11, 379-393.

Peters, R. H., LeVasseur, M. E. y Chandler, R. K. (2004). Correctional treatment for co-occurring disorders: results of a national survey. Behavioral Sciences and The Law, 22, 563-584.

Pettinati, H. M., Rukstalis, M. R., Luck, G. J., Volpicelli, J. R. y O'Brien, C. P. (2000). Gender and psychiatric comorbidity: Impact on clinical presentation of alcohol dependence. American Journal on Addictions, 9, 242-252.

Piselli, M., Elisei, S., Murgia, N., Quartesan, R. y Abram, K. M. (2009). Co-occurring psychiatric and substance use disorders among male detainees in Italy. International Journal of Law and Psychiatry, 32, 101-107.

Regier, D. A., Farmer, M. E., Rae, D. S., Locke, B. Z., Keith, S. J., Judd, L. L. y Goodwin, F. K. (1990). Comorbidity of mental disorders with alcohol and other drug abuse. Results from the Epidemiologic Catchment Area (ECA) Study. Jama, 264, 2511-2518.

Reglamento Penitenciario, aprobado por el Real Decreto 190/1996.

Rodríguez-Llera, M.C., Domingo, A., Brugal, M.T., Silva, T.C., SánchezNiubó, A., Torrens, M., et al. (2006). Psychiatric comorbidity in young heroin users. Drug and Alcohol Dependence, 84, 48-55.

Sheehan, D. V., Lecrubier, Y., Sheehan, K. H., Amorim, P., Janavs, J., Weiller, E., Hergueta, T., Baker, R. y Dunbar, G. C. (1998). The Mini-International Neuropsychiatric Interview (M.I.N.I.): the development and validation of a structured diagnostic psychiatric interview for DSM-IV and ICD-10. Journal of Clinical Psychiatry, 59 Suppl 20, 22-33; quiz 34-57.

Simpler, A., y Langhinrichsen-Rohling, J. (2005). Substance use in prison: How much occurs and is it associated with psychopathology? Addiction Research and Theory, 13, 503-511.

Smith, H., Sawyer, D. A., y Way, B. B. (2002). Central New York Psychiatric Center: An approach to the treatment of co-occurring disorders in the New York State correctional 
mental health system. Behavioral Sciences \& the Law, 20,523534.

Swanson, J.W., Holcer, C., Ganju, V. y Jono, R. (1990). Violence and psychiatric disorder in the community. Hospital Community Psychiatry, 4, 101-137.

Swartz, J. A. y Lurigio, A. J. (1999). Psychiatric illness and comorbidity among adult male jail detainees in drug treatment. Psychiatric Services, 50, 1628-1630.

White, M. D., Goldkamp, J. S., y Campbell, S. P. (2006). Co-Occurring Mental IIIness and Substance Abuse in the Criminal Justice System: Some Implications for Local Jurisdictions. The Prison Journal, 86, 301 - 326. 